\title{
Téoros
}

Revue de recherche en tourisme

\section{Tourisme et patrimoine mondial, Sous la direction de Laurent BOURDEAU, Maria GRAVARI-BARBAS et Mike ROBINSON, Collection Patrimoine en mouvement - PAMV, Québec : Presse de l’Université Laval, 2012, 326 pages, ISBN : 978-2-7637-9758-8}

\section{Mathieu Dormaels}

Volume 30, numéro 2, 2011

URI : https://id.erudit.org/iderudit/1012252ar

DOI : https://doi.org/10.7202/1012252ar

Aller au sommaire du numéro

Éditeur(s)

Université du Québec à Montréal

ISSN

0712-8657 (imprimé)

1923-2705 (numérique)

Découvrir la revue

Citer ce compte rendu

Dormaels, M. (2011). Compte rendu de [Tourisme et patrimoine mondial, Sous la direction de Laurent BOURDEAU, Maria GRAVARI-BARBAS et Mike ROBINSON, Collection Patrimoine en mouvement - PAMV, Québec : Presse de l'Université Laval, 2012, 326 pages, ISBN : 978-2-7637-9758-8]. Téoros, 30(2), 129-129. https://doi.org/10.7202/1012252ar d'utilisation que vous pouvez consulter en ligne. 


\section{POUR EN LIRE PLUS}

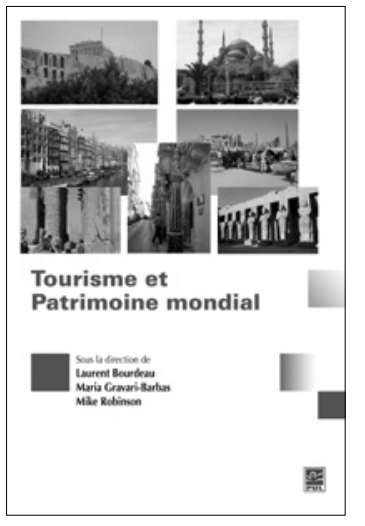

\section{Tourisme et patrimoine mondial}

Sous la direction de Laurent BOURDEAU, Maria GRAVARI-BARBAS et Mike ROBINSON

Collection Patrimoine en mouvement - PAMV

Québec: Presse de l'Université Laval, 2012, 326 pages

ISBN : 978-2-7637-9758-8

La relation entre le tourisme et le patrimoine a fait l'objet de quelques monographies, certaines récentes, qui traitent principalement des avantages, des inconvénients et des techniques de la mise en tourisme d'éléments patrimoniaux. Bien que le patrimoine mondial semble être, entre tous, celui qui induit le plus d'activités touristiques, il n'a pour le moment donné lieu qu'à peu de publications et d'articles dans des revues spécialisées, notamment en français. La parution de cet ouvrage vient donc combler en partie un manque qui est aussi la conséquence de la «jeunesse» de ces études.

Ce livre est constitué de 17 articles sélectionnés parmi les communications présentées au congrès World Heritage and Tourism, Managing for the Local and the Global, organisé par le réseau UNITWIN-UNESCO «Tourisme, Culture, Développement », et qui s'est tenu à Québec en juin 2010. Composé d'articles théoriques et d'étude de cas, il s'adresse bien sûr aux chercheurs, mais aussi aux gestionnaires ou aux habitants des sites inscrits au patrimoine mondial.

Plutôt qu'une série d'exposés de situations, c'est vraiment à une réflexion sur la relation profonde entre tourisme et patrimoine mondial qu'invite le livre. En effet, derrière ces analyses se pose la question de la notion de patrimoine mondial et de son essence. Les auteurs proposent de prendre de la distance avec les perspectives classiques et de dépasser l'antagonisme du site patrimonial et du «tourisme destructeur», pour mettre en évidence une réalité plus complexe. Les différences entre les désormais très nombreux sites inscrits, ainsi que les perceptions et représentations qui constituent la réalité locale des sites, démontrent combien une vision trop simpliste ou trop standard ne peut plus suffire. Par ailleurs, l'ampleur du phénomène semble rendre inévitable le questionnement quant aux impacts sur les populations locales, et pas dans une vision postcoloniale de perversion culturelle, mais bien dans la modification des équilibres entre les groupes sociaux, dans les enjeux de pouvoir et de légitimité pour la réappropriation de ces patrimoines.Les éditeurs du livre posent donc la question d'un type de patrimoine, le patrimoine mondial, dont l'essence même produirait un tourisme "endogène», susceptible sous certaines conditions de devenir même "prescripteur» de patrimoine.

Organisé en trois sections, l'ouvrage interroge trois problématiques majeures pour la compréhension de la relation entre tourisme et patrimoine mondial. La première partie (cinq chapitres) pose la question des différentes valeurs et des différentes significations du patrimoine, et de leurs liens avec les différentes pratiques des lieux, comme touriste et comme résident. La deuxième partie (six chapitres) s'intéresse plus directement aux acteurs et aux stratégies de la «gouvernance patrimoniale» de ces sites. Enfin, la dernière partie (six chapitres) interroge la relation entre l'inscription sur la Liste, ou la reconnaissance par l'UNESCO à un autre titre, et la «labellisation» du patrimoine.

Peut-être ne manque-t-il à ces études qu'une quatrième partie qui aurait questionné la gestion et l'administration du patrimoine mondial au niveau institutionnel, et le futur de cette administration dont il semble bien qu'elle devienne de plus en plus difficile à mesure que la Liste s'agrandit. Cela aurait d'ailleurs rejoint sans doute les interrogations actuelles quant au futur de la Convention concernant la protection du patrimoine mondial, culturel et naturel, à l'aune de ses 40 ans. On regrettera toutefois que le livre ne propose que des articles en français alors que le congrès rassemblait des chercheurs de plusieurs pays et qu'il aurait pu être intéressant de conserver cette diversité, même si l'on comprend bien la complexité d'une telle entreprise.

Il reste que l'ensemble propose une approche prometteuse et jette les bases pour les recherches en cours et à venir, et il est très probable que cet ouvrage devienne une référence en la matière.

Mathieu DORMAELS, Chercheur associé, Chaire de recherche du Canada en patrimoine urbain (ESG-UQAM) 